\title{
Are physiological changes experienced by healthy subjects during acu-TENS associated with acupuncture point sensations?
}

\author{
David T W Yu, ${ }^{1}$ Alice $\mathrm{Y}$ M Jones ${ }^{2}$
}

${ }^{1}$ Department of Rehabilitation Sciences, The Hong Kong Polytechnic University, Hong Kong, China

${ }^{2}$ School of Rehabilitation Sciences, Griffith University, Gold Coast Campus, Gold Coast, Queensland, Australia

\section{Correspondence to}

David Yu, Physiotherapy Department, Queen Elizabeth Hospital, Kowloon, Hong Kong 852, China; yutwd@ha.org.hk

Received 18 July 2013 Accepted 10 October 2013 Published Online First 1 November 2013
To cite: Yu DTW, Jones AYM. Acupunct Med 2014;32: 28-36.

\begin{abstract}
Background Transcutaneous electrical nerve stimulation over acupuncture points (acu-TENS) has been reported to improve clinical outcomes. The objectives of the present study were to investigate whether acupuncture point sensations were experienced during acu-TENS, and whether such sensations were associated with any concomitant changes in autonomic nervous system activity.
\end{abstract}

Methods This study adopted a single-blinded, randomised, controlled trial methodology. A total of 36 healthy subjects were randomly assigned to an experimental group (acu-TENS on right LI4 and LI11 points); control group (acu-TENS to bilateral kneecaps); or placebo group (sham acu-TENS on right LI4 and LI11 points). Heart rate $(H R)$, mean arterial blood pressure (MAP), SD of the NN interval (SDNN) and low frequency to high frequency ratio (LF/HF) were measured before, during and after intervention. The Hong Kong Chinese version of the Massachusetts General Hospital Acupuncture Sensation Scale (C-MMASS) index was used for quantifying the acupuncture point stimulation sensations.

Results The experimental group showed a significant increase in HR (mean (SD) 73.5 (6.3) to 75.9 (6.7) bpm, $p=0.027), \operatorname{MAP}(88.5$ (4.5) to 91.0 (4.1) $\mathrm{mm} \mathrm{Hg}, \mathrm{p}=0.004)$, SDNN (143.36 (8.58) to 153.69 (7.64) $\mathrm{ms}, \mathrm{p}=0.002$ ) and $\mathrm{LF} / \mathrm{HF}$ (1.26 (0.19) to $1.31(0.21), p=0.037)$ during the intervention. The control group showed a significant increase in SDNN (140.21 (8.72) to 143.39 (9.47) ms, $\mathrm{p}=0.009)$ and LF/HF (1.21 (0.09) to $1.23(0.12), p=0.033)$. There were no significant physiological changes in the placebo group. Overall C-MMASS indices for the experimental, control and placebo groups were 3.23 (0.3), $2.14(0.6)$ and 0.29 (0.32), respectively. The between-group difference was statistically significant $(F=139.24, d f=2, p<0.05)$. However, correlation analysis did not support any association between sensation intensity and physiological responses in any groups ( $\gamma$ ranged from -0.36 to 0.25 ).

Conclusions This study showed that 'acupuncture point sensations' were experienced during acu-TENS to LI4 and LI11, but such sensations were not associated with physiological responses induced during the stimulation.

\section{INTRODUCTION}

Complementary treatments are receiving increased attention as a choice for health maintenance. Traditional Chinese Medicine (TCM) practitioners believe acupuncture is able to modulate $q i$ flow and restore the yin-yang balance, and hence health can be maintained. ${ }^{1}{ }^{2}$ In Western medicine, acupuncture concepts have been explained as a regulation of the autonomic nervous system (ANS), revitalising and balancing the body's homeostasis through modulation of the sympathetic and parasympathetic systems. ${ }^{3}{ }^{4}$ Analysis of heart rate variability (HRV), has become a common measurement parameter in many acupuncture studies. ${ }^{5-10}$

While positive effects of acupuncture have been acknowledged by many studies, its associated adverse effects have also been reported. ${ }^{11} 12$ Thus, non-invasive techniques including acupressure ${ }^{13} 14$ and laser acupuncture $^{15}$ have been seen as 'safer' alternatives. Transcutaneous electrical nerve stimulation (TENS) applied over acupuncture points (acu-TENS), has been reported to be effective in reducing dyspnoea in patients with chronic obstructive pulmonary disease (COPD), ${ }^{16}{ }^{17}$ facilitate early haemodynamic recovery after open heart surgery, ${ }^{18}$ relieve labour pain ${ }^{19} 20$ and reduce blood pressure changes in normal healthy subjects. ${ }^{21} 22$ Acu-TENS is 
also associated with an increase in blood $\beta$-endorphin level, ${ }^{17} 23$ a decrease in airway resistance ${ }^{24}$ and is possibly associated with regulation of the ANS. ${ }^{17} 2324$

Comparative investigation of the mechanism of action of acu-TENS and acupuncture continues, but a fundamental criterion for effective acupuncture, 'de $q i$ ', has not been examined during acu-TENS. In TCM terms, de $q i$ is a unique sensation that indicates an appropriate psychophysical response to acupuncture. $^{12}$ In Western medicine, the de qi sensation is thought to indicate stimulation of the ANS via modulation of the cerebrocerebellar limbic system. ${ }^{25} 26$ Based on previous reports on acu-TENS, ${ }^{16}{ }^{17}$ we hypothesise that acu-TENS can induce ANS changes similar to acupuncture; however, whether acupuncture point sensations described as de qi are necessary for the ANS changes elicited during acu-TENS has not been investigated. This study therefore aims to investigate acupuncture point sensations experienced during acu-TENS and whether such sensations are associated with any concomitant changes in ANS activity.

\section{METHODS}

\section{Subjects}

This study adopted a single-blinded (patient-blinded), randomised, controlled trial design. Ethics approval was obtained from the Human Subjects Review Committee of the involved university (Project ID: HSEARS20110510001) and was carried out within the ethical standards set forth in the Helsinki Declaration of 1975. Written consent was obtained from all participants. Normal healthy subjects who were naïve to TENS or acupuncture or acu-TENS and able to read Chinese were recruited. Subjects were excluded if they had a history of cardiopulmonary, neurological and psychological disorders, or if they required any medication for cardiovascular, neurological or psychological conditions within 1 week prior to the study.

\section{Sample size calculation}

Using the analysis and sample size software PASS 2008 (http://www.ncss.com/pass.html), and based on the results of our previous study on de qi measurement, ${ }^{27}$ to achieve a medium effect size, power of 0.8 and produce a 2 -sided $95 \% \mathrm{CI}$ with a width equal to 0.987 and SD of 2.00 , a total sample size of 36 subjects was considered appropriate for the study.

\section{Randomisation procedure}

Subjects were randomly assigned to one of the three groups using sequentially numbered, opaque sealed envelope simple randomisation method. ${ }^{28}$ A total of 12 sheets of paper were marked with 'experimental group', 12 sheets with 'control group' and 12 sheets with 'placebo group', and the sheets were then put into 36 opaque envelopes ( 1 sheet per envelope), which were then sealed. The deck of envelopes was shuffled thoroughly by an independent assistant.
Then, the front of each envelope was marked sequentially with the numbers $1-36$. These envelopes were then placed in numerical order in a plastic container. Each subject was asked to draw an envelope, which was then opened by an independent investigator.

\section{Experimental procedure}

On arrival at the laboratory, subjects were asked to rest for $30 \mathrm{~min}$ in a sitting position to establish a steady cardiopulmonary state. Subjects were instructed to breathe normally, to minimise any effect of respiration on HRV. All measurements were taken between 16:00 and 18:00 to minimise the circadian effect on the ANS. ${ }^{29}$

Systolic and diastolic blood pressures were taken on the left arm by a digital patient monitor (Mindray PM-8000 Express Patient Monitor, Bio-Medical Electricity, Hamburg, Germany). The mean arterial pressure (MAP) was recorded. Electrocardiogram (ECG) electrodes were applied over left and right clavicles and left upper quadrant of abdomen and then connected to the digital patient monitor device. HR was continuously monitored. The ECG signals were analysed by Chart 5 Pro for Windows. The ECG signals were transferred to the PowerLab 16/30 (ADInstruments Pty Ltd., New South Wales, Australia) for data acquisition and analysis of the HRV.

MAP, HR and HRV parameters were measured at $5 \mathrm{~min}$ before the intervention, every $5 \mathrm{~min}$ for $45 \mathrm{~min}$ during the intervention and at the 5 th minute after the stimulation. Data recorded during the $45 \mathrm{~min}$ intervention were averaged for analysis. Immediately after the intervention, subjects were asked to complete the Hong Kong Chinese version of the Massachusetts General Hospital Acupuncture Sensation Scale (C-MMASS) ${ }^{27}$ to quantify the acupuncture point stimulation sensations.

\section{HRV measurement}

The HRV was analysed in accordance with the recommendations of the Task Force of the European Society of Cardiology and the North American Society of Pacing and Electrophysiology. ${ }^{30}$ For frequency domain analysis, power spectral analysis was performed by the Fast Fourier Transformation technique. ${ }^{29}{ }^{30}$ Low frequency (LF), defined as the power between 0.03 and $0.15 \mathrm{~Hz}$, mainly represents sympathetic modulation; high frequency (HF), defined as the power between 0.15 and $0.5 \mathrm{~Hz}$, is regarded as an index of vagal (parasympathetic) modulation; the ratio $\mathrm{LF} / \mathrm{HF}$ reflects the global sympathovagal balance of the body. ${ }^{30}{ }^{31}$ For the time domain analysis, the recorded $\mathrm{SD}$ of the $\mathrm{NN}$ interval (SDNN) is referred to as the global index of the HRV. ${ }^{29} 3031$

\section{Point location and preparation}

The locations of the right LI4 and LI11 points and the apex of patella were identified by a trained physiotherapist. Isopropyl alcohol was applied for skin 
preparation after locating the points. Two selfadhesive ECG electrodes (F-601 SKINTACT) were then placed over the acupuncture points and attached to a dual channel portable TENS unit $(120 \mathrm{z}$ Dual Channel TENS, EMS Physio Ltd, Oxfordshire, UK). The pulse frequency and pulse width of the TENS were set at $2 \mathrm{~Hz}$ and $200 \mathrm{~ms}$, respectively, with the intensity adjusted to the subject's maximum tolerable level short of muscle contraction. Total duration of stimulation was 45 min. ${ }^{17} 2324$

\section{Intervention protocols}

All interventions were conducted in the sitting position. For the experimental group, subjects received acu-TENS over the right LI4 and LI11 points; for the control group, subjects received TENS over the apex of bilateral kneecaps; for the placebo group, subjects received the same protocol as the experimental group and TENS but the points were covered with nonconducting plastics of the same size as the TENS electrodes, such that no electrical current would pass through the skin. Subjects were informed that they might or might not feel the electrical stimulation depending on the pulse frequency used.

\section{Modified Massachusetts General Hospital Acupuncture Sensation Scale, Chinese version}

The C-MMASS consists of 12 descriptors of sensation, including soreness, aching, deep pressure, heaviness, fullness/distension, tingling, numbness, dull pain, warmth, cold, throbbing, plus a blank supplementary row left for subjects to describe their own perceptions. ${ }^{27}$ Each descriptor is presented on a $10-\mathrm{cm}$ bar, anchored with numerical scores $0-10$ and with the words 'none', 'mild', 'moderate' and 'severe' spaced evenly along the continuum. Immediately after the 45 -min intervention, subjects were asked to quantify the sensations experienced by rating the intensity for each descriptor. The C-MMASS index was then calculated $^{27}$ and was used to quantify the overall intensity of the acupuncture sensations experienced.

\section{Statistical analysis}

All statistical analyses were conducted using the Statistical Package for Social Sciences (SPSS) V.17.0 for Windows. Demographic and baseline data were compared among the three groups using one-way analysis of variance (ANOVA). The within-group change in HR, MAP, LF/HF, SDNN and the C-MMASS index of the groups at before, during and after intervention were analysed by one-way repeated ANOVA. Between-group differences were analysed by one-way ANOVA with post hoc pairwise comparison and least significant difference (LSD) adjustment. The null hypothesis was rejected if the calculated $\mathrm{F}$ ratio exceeds the critical value with $\mathrm{p}<0.05$. An exploratory correlation analysis using Spearman's correlation was performed between the overall C-MMASS index, mean MAP, HR, SDNN and LF/HF in each group. The level of significance was set at 0.05 for all analyses.

\section{RESULTS}

In all, 36 participants ( 22 men and 14 women) were recruited for this study (mean (SD): age 32.8 (2.1) years).

\section{Blood pressure changes}

The MAP increased significantly from 88.5 (4.5) $\mathrm{mm} \mathrm{Hg}$ to 91.0 (4.1) $\mathrm{mm} \mathrm{Hg}$ during acu-TENS in the experimental group $(\mathrm{p}=0.004)$; at $5 \mathrm{~min}$ after intervention, the MAP returned to $89.3(4.6) \mathrm{mm} \mathrm{Hg}$ and was still slightly higher than baseline but did not reach statistical significance $(p=0.898)$. There was no significant change in MAP for the control (from 88.7 (5.9) $\mathrm{mm} \mathrm{Hg}$ to $89.6(5.8) \mathrm{mm} \mathrm{Hg})(\mathrm{p}=0.315)$ or the placebo groups $(86.6 \quad(5.7) \mathrm{mm} \mathrm{Hg}$ to 87.1 $(5.4) \mathrm{mm} \mathrm{Hg})(\mathrm{p}=0.3)$ during the stimulation (figure 1).

\section{Heart rate changes}

The HR increased significantly from 73.5 (6.3) bpm to 75.9 (6.7) bpm during acu-TENS in the experimental group $(p=0.027)$ and gradually returned to baseline $(74.6(8.4) \mathrm{bpm})$ post stimulation. There was also a slight increase in HR in the control group from 73.0 (4.5) bpm to $74.2(3.7) \mathrm{bpm}$ during the stimulation period, but this was not statistically significant $(p=0.57)$; the HR returned to baseline at 5 min after the stimulation. For the placebo group, there was no significant changes in HR (from 72.1 (6.9) bpm to

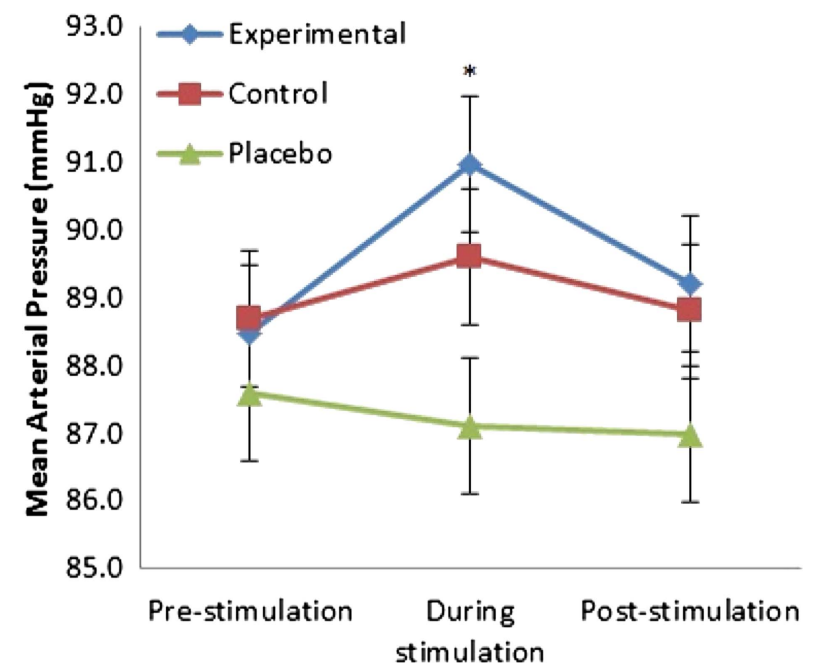

Figure 1 Changes in mean arterial pressure at various measurement timepoints in the three groups. Experimental group: transcutaneous electrical nerve stimulation over acupuncture points (acu-TENS) to right LI4 and LI11 points; control group: TENS to bilateral kneecaps; placebo group: TENS applied to non-conductive plastic films on the right LI4 and LI11 points. *Difference of mean arterial pressure between prestimulation and during stimulation in the experimental group $(p<0.05)$. 


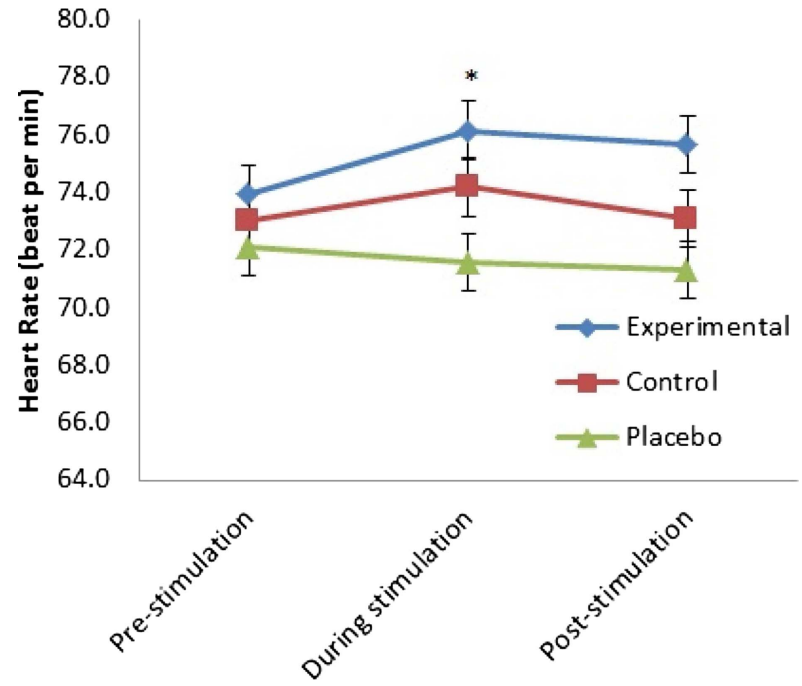

Figure 2 Heart rate changes at various measurement timepoints in the three groups. Experimental group: transcutaneous electrical nerve stimulation over acupuncture points (acu-TENS) to right LI4 and LI11 points; control group: TENS to bilateral kneecaps; placebo group: TENS applied to non-conductive plastic films on the right LI4 and LI11 points. *Difference of heart rate between prestimulation and during stimulation in the experimental group $(p<0.05)$.

71.6 (4.7) bpm) throughout the stimulation period $(\mathrm{p}=0.831)$ (figure 2).

\section{SDNN changes}

The SDNN increased from 143.36 (8.58) $\mathrm{ms}$ to 153.69 (7.64) $\mathrm{ms}$ in the experimental group during stimulation $(\mathrm{p}=0.002)$; at $5 \mathrm{~min}$ post stimulation, it remained higher than the baseline value by $6.31 \mathrm{~ms}$ $(\mathrm{p}=0.037)$. For the control group, the SDNN increased from 140.21 (8.72) ms to 143.39 (9.47) ms during the stimulation $(\mathrm{p}=0.009)$; at $5 \mathrm{~min}$ post stimulation, it was $2.46 \mathrm{~ms}$ higher than the baseline but the change was not statistically significant $(p=0.068)$. For the placebo group, there was no significant change in SDNN during or after the stimulation (144.38 (10.19) $\mathrm{ms}$ to $144.23 \quad(10.23) \mathrm{ms})$, (figure 3). A significant between-group difference in the SDNN during the stimulation was found among the three groups $(\mathrm{F}=4.06, \mathrm{df}=2, \mathrm{p}=0.027)$. Post hoc analysis with LSD adjustment showed there was a significant difference in the SDNN between the experimental and control groups ( $p=0.013$, CI 2.3 to 18.3) and between the experimental and placebo groups $(p=0.029$, CI 0.97 to 17.0$)$. There was also a significant main effect for acu-TENS on SDNN $(\mathrm{F}=19.068$, $\mathrm{p}<0.05)$ and a significant interaction effect between acu-TENS and the period of stimulation $(\mathrm{F}=7.91$, $\mathrm{p}<0.05$ ).

\section{LF/HF changes}

The LF/HF increased from $1.26(0.19)$ to $1.32(0.21)$ during acu-TENS $(p=0.019)$ and at 5 min after the

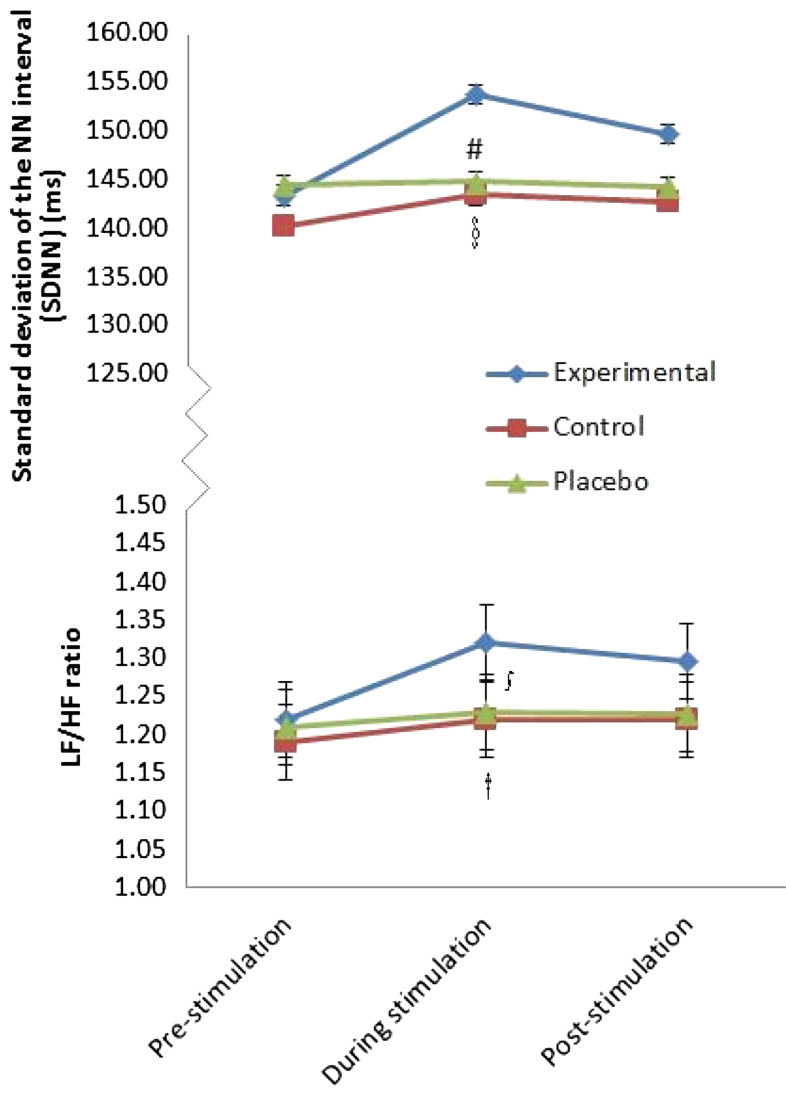

Figure 3 SDNN and LF/HF changes at various measurement timepoints in the three groups. Experimental group: transcutaneous electrical nerve stimulation over acupuncture points (acu-TENS) to right LI4 and LI11 points; control group: TENS to bilateral kneecaps; placebo group: TENS applied non-conductive plastic films on the right LI4 and LI11 points. \#Difference of SDNN between the experimental group and the control group during acu-TENS $(p<0.05)$. §Difference of SDNN between the experimental group and the placebo group during acu-TENS $(p<0.05)$. JDifference of LF/HF between the experimental group and the control group during acu-TENS $(p<0.05)$. tDifference of LF/HF between the experimental group and the placebo group during acu-TENS $(p<0.05)$. HF, high frequency; LF, low frequency; SDNN, standard deviation of the NN interval.

stimulation $(1.30 \quad(0.17))(p=0.037)$. There was a small but statistically significant increase in LF/HF in the control group during the stimulation period (from $1.21(0.09)$ to $1.23(0.12))(\mathrm{p}=0.033)$. This ratio returned to baseline $5 \mathrm{~min}$ after the stimulation. Changes in this ratio were not significant in the placebo group, where the $\mathrm{LF} / \mathrm{HF}$ remained at 1.23 (0.11) throughout the intervention (figure 3). A significant between-group difference in the LF/HF was found during the stimulation period $(\mathrm{F}=11.25, \mathrm{df}=2$, $\mathrm{p}=0.03$ ). Post hoc analysis with LSD adjustment showed there was a significant difference in the LF/ HF between the experimental and control groups $(\mathrm{p}=0.02$, CI 0.45 to 2.2$)$ and between the experimental and placebo groups $(\mathrm{p}=0.017$, CI 0.4 to 2.3$)$. Furthermore, there was a significant primary effect for acu-TENS on LF/HF $(\mathrm{F}=2.73, \mathrm{p}=0.044)$ while there 
was no significant interaction effect between acu-TENS and the period of stimulation $(\mathrm{F}=0.411$, $\mathrm{p}=0.72$ ).

\section{Intensity of acupuncture point sensations perceived during intervention}

There was a significant difference in the C-MMASS index among the three groups $(\mathrm{F}=139.24, \mathrm{df}=2$, $\mathrm{p}<0.05$ ); with the overall C-MMASS indices of the experimental, control and placebo groups being 3.23 (0.3), 2.14 (0.6) and 0.29 (0.32), respectively. Post hoc analysis with LSD adjustment showed that there was a significant difference in the C-MMASS index between the experimental and control groups $(\mathrm{p}<0.05$, CI 0.72 to 1.45$)$ and between the experimental and placebo groups $(\mathrm{p}<0.05$, CI 2.57 to $3.30)$. In the experimental group, the three descriptors recorded with highest ratings were throbbing, soreness and fullness/distension. For the control group, the three strongest sensations recorded were tingling, throbbing and numbness. For the placebo group, the strongest sensation recorded was tingling. No subject opted to add any new descriptors in the blank row provided (figure 4).

\section{Association between C-MMASS score and the physiological responses}

Correlation analysis between the overall C-MMASS index score and HR, MAP, SDNN and LF/HF revealed a very low association between the intensity of sensations and any of the recorded physiological responses in all groups (table 1).

\section{DISCUSSION}

This is the first study to report a relationship between acu-TENS and acupuncture point sensations, similar to de qi reported in the literature. ${ }^{3} 3233$ The results show that acupuncture point sensations were experienced by our healthy cohort and were strongest during acu-TENS, compared to non-acupuncture point and placebo stimulations.

De $q i$ is believed to be an indispensable component of effective acupuncture. ${ }^{3}$ Previous studies have shown that de qi sensation is associated with regulation of blood flow, ${ }^{34-38}$ changes in neuronal activity in different areas of the brain, ${ }^{39-41}$ changes in ANS activities $^{42-48}$ and an increase in pain threshold. ${ }^{49} \mathrm{~A}$ survey conducted by the British Acupuncture Council on 574 members revealed that $87 \%$ of acupuncturists would aim to attain de qi during acupuncture. ${ }^{50}$ Our previous work demonstrated that needle electroacupuncture to LI4 and LI11 increases sympathetic discharge, which was strongly associated with acupuncture point sensations. ${ }^{42}$ Insertion of the needle into the acupuncture points, together with the needle-twirling manoeuvre, lead to muscle tension, trembling, twitching and spasm; this in turn stimulates muscle proprioceptors and gives rise to distinct needle sensations. ${ }^{51}$ Such sensory inputs modulate ANS activities by regulating the brain activity through deactivation of the cerebrocerebellar limbic systems. ${ }^{34}$ TENS, however, stimulates the human body through a different mechanism. TENS induces cutaneous stimulation through burst train mode, low pulse frequency, electrical activity. ${ }^{22} 52$ Application of TENS to acupuncture points may induce acupuncture point sensations

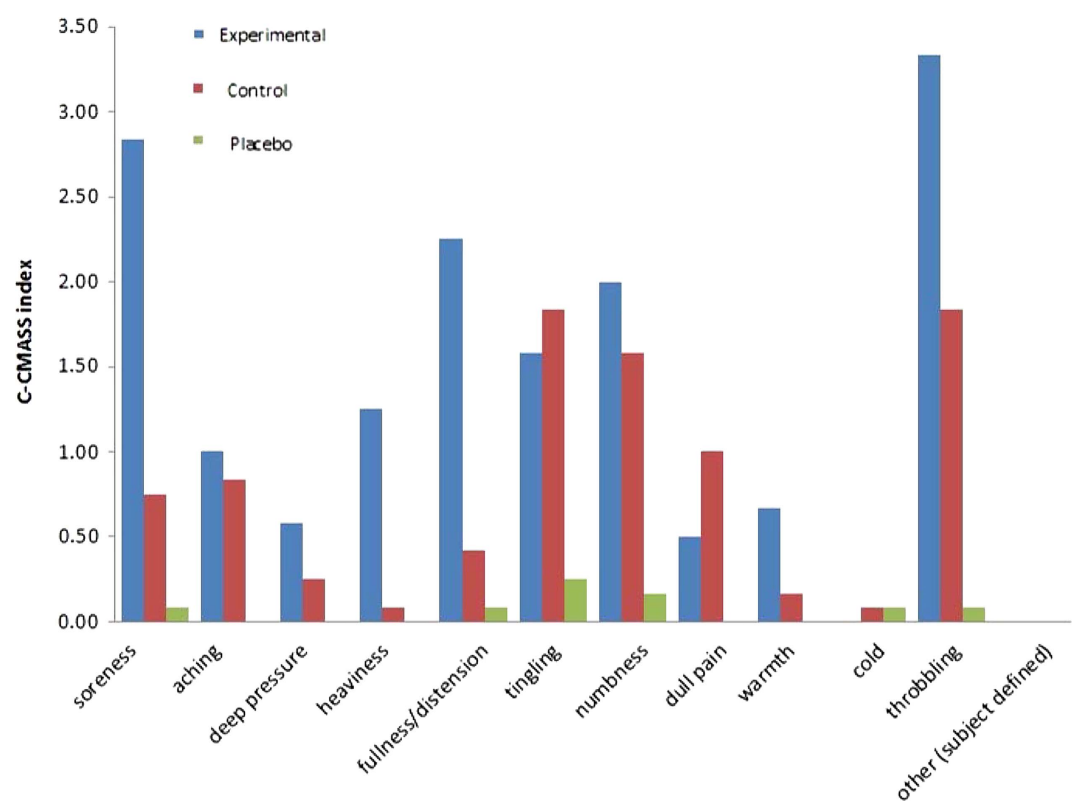

Figure 4 Intensity of sensations expressed by C-MMASS index score reported by different groups. Experimental group: transcutaneous electrical nerve stimulation over acupuncture points (acu-TENS) to right LI4 and LI11 points; control group: TENS to bilateral kneecaps; placebo group: TENS applied non-conductive plastic films on the right LI4 and LI11 points. C-MMASS, Modified Massachusetts General Hospital Acupuncture Sensation Scale, Chinese version. 
Table 1 Correlation between C-MMASS and the physiological responses

\begin{tabular}{llllr}
\hline C-MMASS score & MAP & HR & SDNN & LF/HF \\
\hline Experimental group & $-0.16(p=0.62)$ & $0.08(p=0.81)$ & $-0.32(p=0.32)$ & $-0.06(p=0.85)$ \\
Control group & $-0.42(p=0.17)$ & $-0.06(p=0.86)$ & $-0.36(p=0.6)$ & $-0.07(p=0.12)$ \\
Placebo group & $-0.26(p=0.41)$ & $0.25(p=0.15)$ & $0.08(p=0.81)$ & $0.09(p=0.76)$ \\
\hline
\end{tabular}

Experimental group: acu-TENS to right LI4 and LI11 points; control group: TENS to bilateral kneecaps; placebo group: TENS applied to non-conductive plastic films on the right LI4 and LI11 points.

Acu-TENS, transcutaneous electrical nerve stimulation over acupuncture points; C-MMASS, Modified Massachusetts General Hospital Acupuncture Sensation Scale, Chinese version; HF, High frequency; HR, heart rate; LF, low frequency; MAP, mean arterial blood pressure; SDNN, SD of the NN interval.

through electrical stimulation of the particular acupuncture point.

The results of this current study demonstrate that subjects in the experimental group experienced strong sensations of throbbing, soreness, fullness and distension, similar to the sensations described during needle acupuncture. The C-MMASS score was also reported to be highest in the experimental group, moderate in the control group and minimal in the placebo group. Anatomically, acupuncture points are areas with a high density of nerve endings and lower electrical impedance compared to other tissues. ${ }^{53-56}$ It is possible that the 'sensation' experienced by subjects in the experimental and control groups was due to electrical current stimulation. The lower C-MMASS score recorded in the control group may be a consequence of a lower density of nerve endings in the patella region, eliciting weaker sensations compared to stimulation of actual acupuncture points. However, the acupuncture point sensations reported in the control group were different from those reported by the acu-TENS group. The dominant sensation reported by our control group was tingling, numbness and throbbing. This could be sensation of electrical stimulation rather than de qi. It is therefore our postulation that the 'acupuncture point effect' could at least, in part, account for the high C-MMASS score recorded in our subjects in the experimental group, and not just have arisen as a consequence of current stimulation. However, this study also suggests that the acupuncture point sensations, with descriptive features similar to de $q i$, are not associated with ANS changes induced during acu-TENS.

It is interesting to note that although there should be no electrical stimulation occurring in the placebo group, some subjects still reported mild sensations of tingling, soreness, fullness/distension and throbbing during the intervention. A previous report has also shown that placebo acu-TENS over Dingchuan (EX-B1) could increase forced expiratory volume in 1 second $\left(\mathrm{FEV}_{1}\right)$, reduce respiratory rate and dyspnoea score in patients with COPD, ${ }^{17}$ and reduce airway resistance in healthy individuals after exercise. ${ }^{24}$ Our speculation is that despite our novel placebo method of impeded electrical current to the skin, tactile sensations over the site of electrode placement were still experienced. It is possible that when one is focused on a certain body area, one could become aware of spontaneous activity of motorneurons or peripheral sensory afferents. ${ }^{57}$ Furthermore, there is evidence to suggest that complex medical interventions or medical devices have high placebo effects. ${ }^{58}$ The intervention procedures in this study required complex setup and frequent subject-practitioner contacts; therefore, the subjects in the placebo group may have a high expectation that contributed to the slight distinct sensations experienced.

In contrast to other studies which showed that TENS decreased sympathetic tone by providing powerful analgesic effects through the release of opioid peptides in the central nervous system, ${ }^{23}$ 59-61 our study demonstrated an increase in sympathetic discharge when acu-TENS was applied to LI4 and LI11. It may be possible that acu-TENS has a bidirectional effect when acupuncture points are stimulated; as our subjects were healthy individuals, they did not require modulation of the parasympathetic tone to induce a calming effect. Another explanation for the increase in sympathetic activities induced is that, as LI4 and LI11 are located in the Large Intestine meridian-believed to be the sympathetic division of the ANS, ${ }^{62}$ it is possible that stimulation of the acupuncture points on the yang meridian will increase the sympathetic discharge. Furthermore, it was previously reported that naturally occurring sympathetic action potentials occur in bursts trains. ${ }^{62}$ A burst mode TENS with the intensity at the maximal tolerable level was used in this current study; this may have facilitated sympathetic discharge. This may also explain why when TENS was applied to non-acupuncture points, an increase in sympathetic activities was also demonstrated although to a much lesser extent when compared with acu-TENS.

\section{Limitations of the study}

One limitation of our study is the small sample size; however, our data have produced a satisfactory medium effect size and thus we consider our findings to be valid.

If stimulation were applied to bilateral LI4 and LI11, the acupuncture point stimulation effect could be stronger. However, we were mindful to prevent occlusion of the circulation to the left forearm arm by the blood pressure cuff, especially during the inflation 
phase, as it may consequently perturb the fidelity of the acu-TENS effects on the acupuncture points.

Lastly, direct measurement of sympathetic activities such as muscle sympathetic activity and evaluation of the plasma catecholamine level ${ }^{63}$ could help to provide more concrete evidence to demonstrate the association between acu-TENS and sympathetic outflow.

\section{CONCLUSIONS}

This is the first study to investigate the relationship between acupuncture point sensations and acu-TENS. This study showed that acupuncture point sensations were experienced during acu-TENS to LI4 and LI11; however, as the intensity of the sensations was not correlated with the physiological responses induced, we conclude that acupuncture point sensations are not associated with the physiological responses evoked during acu-TENS.

\section{Summary points}

- When using transcutaneous electrical nerve stimulation (TENS), the relationship between sensation and physiological response is unknown.

- We compared TENS at acupuncture points with TENS at a control site and placebo TENS.

- Real TENS generated physiological responses, but these were not correlated to sensation.

Acknowledgements The authors would like to thank all the subjects who participated in this study.

Contributors All authors were involved in the study design. TWDY and AYMJ were responsible for the conduct of the study and drafting the manuscript. TWDY performed statistical analysis.

\section{Competing interests None.}

Ethics approval Human Subjects Review Committee of the Hong Kong Polytechnic University (Project ID: HSEARS20110510001).

Provenance and peer review Not commissioned; externally peer reviewed.

\section{REFERENCES}

1 Kaptchuk TJ. Acupuncture: theory, efficacy, and practice. Ann Intern Med 2002;136:374-83.

2 Vickers A, Wilson P, Kleijnen J. Acupuncture. Qual Saf Health Care 2002;11:92-7.

3 Kong J, Bollub R, Huang T, et al. Acupuncture De Qi, from qualitative history to quantitative measurement. J Altern Complement Med 2007;13:1059-70.

4 Lin JG, Chen WL. Acupuncture analgesia: a review of its mechanisms of actions. Am J Chin Med 1994;36:635-45.

5 Haker E, Egekvist H, Bjerring P. Effect of sensory stimulation (acupuncture) on sympathetic and parasympathetic activities in healthy subjects. J Auton Nerv Syst 2000;79:52-9.
6 Chang CH, Huang JL, Ting CT, et al. Atropine-induced HRV alteration is not amended by electroacupuncture on Zusanli. Am J Chin Med 2005;33:307-14.

7 Li Z, Wang C, Mak AF, et al. Effects of acupuncture on heart rate variability in normal subjects under fatigue and non-fatigue state. Eur J Appl Physiol 2005;94:633-40.

8 Backer M, Grossman P, Schneider J, et al. Acupuncture in migraine: investigation of autonomic effects. Clin J Pain. 2008;24:106-15.

9 Jeong HS, Yang CS, Nam JS, et al. Short time effect of caffeine on heart rate variability and the effect of acupuncture at Neiguan (PC6): a randomized double blind pilot study. Korean J Orient Int Med 2008;29:778-86.

10 Park SU, Jung WS, Moon SK, et al. Effects of acupuncture on autonomic nervous system in normal subjects under mental stress. J Korean Oriental Med 2008;29:107-15.

11 Lao L, Hamilton GR, Fu J, et al. Is acupuncture safe? A systematic review of case reports. Altern Ther Health Med 2003;9:72-83.

12 White A, Hayhoe S, Hart A, et al. Adverse events following acupuncture: prospective survey of 32000 consultations with doctors and physiotherapists. Acupunct Med 2001;19:84-92.

13 Arai YC, Ushida T, Osuga T, et al. The effect of acupressure at the Extra 1 point on subjective and autonomic responses to needle insertion. Anesth Analg 2008;107:661-4.

14 Arai YC, Ushida T, Matsubara T, et al. The influence of acupressure at Extra 1 acupuncture point on the spectral entropy of the EEG and the LF/HF ratio of heart rate variability. Evid Based Complement Altern Med 2011;2011: 503698. doi:10.1093/ecam/nen061

15 Gruber W, Eber E, Malle-Scheid D, et al. Laser acupuncture in children and adolescents with exercise induced asthma. Thorax 2002;57:222-5.

16 Lau KSL, Jones AYM. A single session of Acu-TENS increases FEV1 and reduces dyspnoea in patients with chronic obstructive pulmonary disease: a randomized, placebo-controlled trial. Aust J Physiother 2008;54:179-84.

17 Ngai SPC, Jones AYM, Hui-Chan CWY, et al. Acute effects of Acu-TENS on FEV1 and blood $\beta$-endorphin level in chronic obstructive pulmonary disease. Altern Ther 2011;17:8-13.

$18 \mathrm{Ng}$ MCS, Jones AYM, Cheng LC. The role of Acu-TENS in hemodynamic recovery after open-heart surgery. eCAM 2011; 2011:301974. doi:10.1093/ecam/neq015

19 Chao AS, Chao A, Wang TH, et al. Pain relief by applying transcutaneous electrical nerve stimulation (TENS) on acupuncture points during the first stage of labor: a randomized double-blind placebo-controlled trial. Pain 2007;127:214-20.

20 Peng T, Li ST, Zhou SF, et al. Transcutaneous electrical nerve stimulation on acupoints relieves labor pain: a non-randomized controlled study. Chin J Integr Med 2010;16:234-8.

21 Zhang J, Ng D, Sau A. Effects of electrical stimulation of acupuncture points on blood pressure. J Chiropr Med 2009;8:9-14.

22 Jones AYM, Kwan YL, Leung NTF, et al. Electrical stimulation of acupuncture points and blood pressure responses to postural changes: a pilot study. Am J Crit Care 2011;20:e67-74.

23 Ngai SPC, Jones AYM, Hui-Chan CW, et al. Effect of 4 weeks of Acu-TENS on functional capacity and beta-endorphin level in subjects with chronic obstructive pulmonary disease: a randomized controlled trial. Respir Physiol Neurobiol 2010;173:29-36. 
24 Chan JCK, Wong NNN, Wong DWH, et al. Effect of Acu-TENS on post exercise airway resistance in healthy individuals. H K Physiother J 2012;30:29-35.

25 Huang W, Pach D, Napadow V, et al. Characterizing acupuncture stimuli using brain imaging with fMRI-a systematic review and meta-analysis of the literature. PLoS ONE 2012;7:e32960.

26 Ueyama T. Emotion, amygdala, and autonomic nervous system. Brain Nerve 2012;64:1113-19.

27 Yu DTW, Jones AYM, Pang MYC. Development and validation of the Chinese version of the Massachusetts General Hospital Acupuncture Sensation Scale: an exploratory and methodological study. Acupunct Med 2012;30:214-21.

28 Altman DG, Schulz KF. Statistics notes: concealing treatment allocation in randomised trials. BMJ 2001;323:446-7.

29 Agelink MW, Malessa R, Baumann B, et al. Standardized tests of heart rate variability: normal ranges obtained from 309 healthy humans, and effects of age, gender, and heart rate. Clin Auton Res 2001;11:99-108.

30 Task Force of the European Society of Cardiology and the North American Society of Pacing and Electrophysiology. Heart rate variability. Standards of measurement, physiological interpretation, and clinical use. Circulation 1996;93:1043-65.

31 Sztajzel T. Heart rate variability: a noninvasive electrocardiographic method to measure the autonomic nervous system. Swiss Med Wkly 2004;134:514-22.

32 Hui KKS, Nixon EE, Vangel MG, et al. Characterization of the "deqi" response in acupuncture. BMC Complement Altern Med 2007;7:33.

33 Wang KM, Yao SM, Xian YL, et al. A study of the receptive field of acupoints and the relationship between characteristics of needling sensation and groups of afferent fibres. Sci Sin $1985 ; 28: 963-71$.

34 Huang T, Yang LY, Zhang WB, et al. Observation of microvascular perfusion in the Hegu (LI4) acupoint area after deqi acupuncture at Quchi (LI11) acupoint using speckle laser blood flow scanning technology. J Evid Based Complement Altern Med 2012;2012:604590. doi:10.1155/2012/604590

35 Sandberg M, Lundeeberg T, Lindberg LG, et al. Effects of acupuncture on skin and muscle blood flow in healthy subjects. Eur J Appl Physiol 2003;90:114-9.

36 Sandberg M, Larsson B, Lindberg LG, et al. Different patterns of blood flow response in the trapezius muscle following needle stimulation (acupuncture) between healthy subjects and patients with fibromyalgia and work-related trapezius myalgia. Eur J Pain 2005;9:497-510.

37 Kuo TC, Chen ZS, Cgen CH, et al. The physiological effect of De Qi during acupuncture. J Health Sci 2004; 50:336-42.

38 Hui KKS, Liu J, Marina O, et al. The integrated response of the human cerebro-cerebellar and limbic systems to acupuncture stimulation at ST 36 as evidenced by fMRI. NeuroImage 2005;27:479-96.

39 Napadow V, Dhond RP, Kim J, et al. Brain encoding of acupuncture sensation-Coupling on-line rating with fMRI. NeuroImage 2009;47:1055-65.

40 Hsieh JC, Tu CH, Chen MC, et al. Activation of the hypothalamus characterizes the acupuncture stimulation at the analgesic point in human: a positron emission tomography study. Neurosci Lett 2001;307:105-8.

41 Napadow V, Kettner N, Liu J, et al. Hypothalamus and amygdala response to acupuncture stimuli in carpal tunnel syndrome. Pain 2007;130:254-66.
42 Yu DTWD, Jones AYM. Physiological changes associated with deqi during electroacupuncture to LI4 and LI11- a randomized placebo-control trial. Acup Med 2013;31:143-50.

43 Haker E, Egekvist H, Bjerring P. Effects of sensory stimulation (acupuncture) on sympathetic and parasympathetic activities in healthy subjects. J Autonom Ner Syst 2000;79:52-9.

44 Saki S, Hori E, Umeno K, et al. Specific acupuncture sensation correlates with EEGs and autonomic changes in human subjects. Auton Neurosci-Basic Clin 2007;133:158-69.

45 Li ZY, Jiao K, Chen M, et al. Effect of magnitopuncture on sympathetic and parasympathetic nerve activities in healthy drivers-assessment by power spectrum analysis of heart rate variability. Eur J Appl Physio 2003;88:404-10.

46 Hsu CC, Weng CS, Liu TS, et al. Effects of electrical acupuncture on acupoint BL15 evaluated in terms of heart rate variability, pulse rate variability and skin conductance response. Am J Chin Med 2006;34:23-36.

47 Knardahl S, Elam M, Olausson B, et al. Sympathetic nerve activity after acupuncture in humans. Pain 1998;75:19-25.

48 Kimura K, Masuda K, Wakayama I. Changes in skin blood flow and skin sympathetic nerve activity in response to manual acupuncture stimulation in humans. Am J Chin Med 2006;34:189-96.

49 Choi Y, Cho S, Lee J, et al. P02.116. The relationship between deqi and the effect of acupuncture. BMC Complement Altern Med 2012;12:172.

50 MacPherson $\mathrm{H}$, Thomas $\mathrm{K}$, Walters $\mathrm{S}$, et al. A prospective survey of adverse events and treatment reaction following 34,000 consultants with professional acupuncturists. Acupunct Med 2001;19:93-102.

51 Li AH, Zhang JM, Xie YK. Human acupuncture points mapped in rats are associated with excitable muscle/skin-nerve complexes with enriched nerve endings. Brain Res 2004;1012:154-9.

52 Sluka KA, Walsh D. Transcutaneous electrical nerve stimulation: basic science, mechanisms and clinical effectiveness. J Pain 2003;4:109-21.

53 Gunn CC, Ditchburn FG, King MH, et al. Acupuncture loci. A proposal for their classification according to their relationship to known neural structures. Am J Chin Med 1976;4:183-95.

54 Liu YK, Varela M, Oswald R. The correspondence between some motor points and acupuncture loci. Am J Chin Med 1975;3:347-58.

55 Falk CX, Brich S, Avants SK, et al. Preliminary results of a new method of locating auricular acupuncture points. Acupunct Electrother Res 2000;25:165-77.

56 Egot-Lemaire SJ-P, Ziskin MC. Dielectric properties of human skin at an acupuncture point in the $50-75 \mathrm{GHz}$ frequency range. A pilot study. Bioelectromagnetics 2011;32:360-6.

57 Hagbarth KE, Vallbo AB. Mechanoreceptor activity recorded percutaneously with semi-microelectrodes in human peripheral nerves. Acta Physiol Scand 1967;69:121-2.

58 Paterson C, Dieppe P. Characteristic and incidental (placebo) effects in complex interventions such as acupuncture. BMJ 2005;330:1202-5.

59 Han JS. Acupuncture and endorphins. Neurosci Lett 2004;361:258-61.

60 Chao AS, Chao A, Wang TH. Pain relief by applying transcutaneous electrical nerve stimulation (TENS) on acupuncture points during the first stage of labor: 
a randomized double-blind placebo-controlled trial. Pain 2007;127:214-20.

61 Tjen-A-Looi SC, Li P, Longhurst JC. Medullary substrate and differential cardiovascular responses during stimulation of specific acupoints. Am J Physiol 2004;287: R852-62.
62 Delius W, Hagbarth KE, Hongell A, et al. Manoeuvers affecting sympathetic outflow in human muscle nerves. Acta Phsyiol Scand 1972;84:82-94.

63 Mark AL, Victor RG, Nerhed C, et al. Microneurographic studies of the mechanisms of sympathetic nerve responses to static exercise in human. Circ Res 1985;57:461-9. 
Copyright of Acupuncture in Medicine is the property of BMJ Publishing Group and its content may not be copied or emailed to multiple sites or posted to a listserv without the copyright holder's express written permission. However, users may print, download, or email articles for individual use. 